\title{
Efficacy and safety of single-inhaler extrafine triple therapy versus inhaled corticosteroid plus long-acting beta2 agonist in eastern Asian patients with COPD: the TRIVERSYTI randomised controlled trial
}

Jinping Zheng ${ }^{1}$, Simonetta Baldi ${ }^{2}$, Li Zhao ${ }^{3}$, Huiping Li ${ }^{4}$, Kwan-Ho Lee $^{5}$, Dave Singh ${ }^{6}$, Alberto Papi ${ }^{7}$, Frédérique Grapin ${ }^{2}$, Alessandro Guasconi ${ }^{2}$ and George Georges ${ }^{2^{*}}$ (D)

\begin{abstract}
Background: A single-inhaler extrafine triple combination of beclometasone dipropionate (BDP), formoterol fumarate (FF) and glycopyrronium (G) has been developed for maintenance therapy of chronic obstructive pulmonary disease (COPD). This study evaluated the efficacy and safety of BDP/FF/G in patients in three eastern Asian areas: China, Republic of Korea and Taiwan.

Methods: TRIVERSYTI was a double-blind, randomised, active-controlled, parallel-group study in patients with COPD, post-bronchodilator forced expiratory volume in $1 \mathrm{~s}\left(\mathrm{FEV}_{1}\right)<50 \%$ predicted, $\geq 1$ exacerbation in the previous 12 months, and receiving inhaled maintenance medication. Patients received either extrafine BDP/FF/G 100/6/10 $\mu \mathrm{g}$ via pressurised metered-dose inhaler, or non-extrafine budesonide/formoterol (BUD/FF) 160/4.5 $\mathrm{mg}$ via dry-powder inhaler, both administered as two puffs twice-daily for 24 weeks. The co-primary objectives (analysed in the overall population) were to demonstrate superiority of BDP/FF/G over BUD/FF for change from baseline in pre-dose morning and 2-h post-dose $\mathrm{FEV}_{1}$ at Week 24 (these were analysed as key secondary objectives in the China subgroup). The rate of moderate/severe COPD exacerbations was a secondary endpoint.
\end{abstract}

Results: Of 708 patients randomised, 88.8\% completed. BDP/FF/G was superior to BUD/FF for pre-dose and 2-h post-dose $\mathrm{FEV}_{1}$ at Week 24 [adjusted mean differences $62(95 \% \mathrm{Cl} 38,85) \mathrm{mL}$ and $113(87,140) \mathrm{mL}$; both $p<0.001$ ]. The annualised moderate/severe exacerbation rate was $43 \%$ lower with BDP/FF/G [rate ratio $0.57(95 \% \mathrm{Cl} 0.42,0.77)$; $p<0.001$. Adverse events were reported by $61.1 \%$ and $67.0 \%$ patients with BDP/FF/G and BUD/FF. Results were similar in the China subgroup.

Conclusions: In patients with COPD, $\mathrm{FEV}_{1}<50 \%$ and an exacerbation history despite maintenance therapy, treatment with extrafine BDP/FF/G improved bronchodilation, and was more effective at preventing moderate/severe COPD exacerbations than BUD/FF.

Trial registration CFDA CTR20160507 (registered 7 Nov 2016, http://www.chinadrugtrials.org.cn/index.html).

\footnotetext{
*Correspondence: george.georges@chiesi.com

${ }^{2}$ Global Clinical Development, Chiesi Farmaceutici SpA, Largo Belloli,

$11 \backslash \mathrm{a}, 43122$ Parma, Italy

Full list of author information is available at the end of the article
}

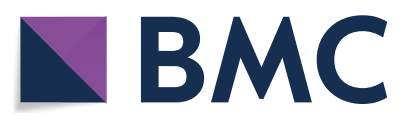

(c) The Author(s) 2021. Open Access This article is licensed under a Creative Commons Attribution 4.0 International License, which permits use, sharing, adaptation, distribution and reproduction in any medium or format, as long as you give appropriate credit to the original author(s) and the source, provide a link to the Creative Commons licence, and indicate if changes were made. The images or other third party material in this article are included in the article's Creative Commons licence, unless indicated otherwise in a credit line to the material. If material is not included in the article's Creative Commons licence and your intended use is not permitted by statutory regulation or exceeds the permitted use, you will need to obtain permission directly from the copyright holder. To view a copy of this licence, visit http://creativecommons.org/licenses/by/4.0/. The Creative Commons Public Domain Dedication waiver (http://creativeco mmons.org/publicdomain/zero/1.0/) applies to the data made available in this article, unless otherwise stated in a credit line to the data. 
Keywords: Triple inhalation therapy, Chronic obstructive pulmonary disease, Chronic bronchitis, Airway obstruction, Extrafine

\section{Background}

Pharmacologic management of chronic obstructive pulmonary disease (COPD) aims to reduce current symptoms and future exacerbation risk [1]. 'Triple therapy' of an inhaled corticosteroid (ICS), a long-acting $\beta_{2^{-}}$ agonist (LABA) and a long-acting muscarinic antagonist (LAMA) is an option for patients with COPD who exacerbate despite ICS/LABA or LABA/LAMA, or who experience symptoms with ICS/LABA [1]. Triple therapy can be administered as ICS/LABA in one inhaler and LAMA in a second, often with different design, instructions for use and dosing regimen; single-inhaler triple therapies simplify this regime, and potentially optimise adherence and persistence. One of these is extrafine beclometasone dipropionate (BDP), formoterol fumarate (FF) and glycopyrronium (G). Extrafine particles (i.e., mass median aerodynamic diameter $<2 \mu \mathrm{m}$ ) are more able to consistently reach small airways than non-extrafine particles [2], enhancing delivery to these airways, a major site of airflow obstruction in COPD [3, 4], with lower oropharyngeal deposition [5].

Three prior 52-week studies have evaluated BDP/FF/G in COPD: BDP/FF/G reduced the rate of COPD exacerbations by $23 \%$ versus BDP/FF in TRILOGY [6], by $20 \%$ versus tiotropium in TRINITY [7], and by $15 \%$ versus indacaterol/glycopyrronium in TRIBUTE [8]. All three studies were conducted in predominantly Caucasian populations. There is high prevalence of COPD in many countries in eastern Asia (13.6\% in China [9]), so new therapeutic options are necessary in these countries. In addition, factors such as ethnicity, environment, education, nutrition and lifestyle may contribute to a differential response to pharmacotherapy. We therefore evaluated the efficacy and safety of BDP/FF/G in patients with COPD in three eastern Asian areas: China, Republic of Korea and Taiwan. To support regulatory approval of BDP/FF/G in China, the study was powered both for the overall population and for the subgroup of patients recruited at sites in China. Furthermore, although the primary analyses were for the overall population, the study's key secondary objectives were analysed in the China subgroup, and all data are presented both overall and for the China subgroup.

\section{Methods}

Trial design

TRIVERSYTI was a multinational, double-blind, doubledummy, randomised, active-controlled, parallel-group study. Eligible patients were aged $\geq 40$ years, diagnosed with COPD $\geq 12$ months, current or ex-smokers ( $\geq 10$ pack-years), post-bronchodilator forced expiratory volume in $1 \mathrm{~s}\left(\mathrm{FEV}_{1}\right)<50 \%$ predicted, $\geq 1$ exacerbation in the previous 12 months, and had been receiving therapy for $\geq 2$ months with ICS/LABA, ICS/LAMA, LABA/ LAMA, LAMA, or LABA (but not ICS/LABA/LAMA). Main exclusion criteria were: diagnosis of asthma; COPD exacerbation in the four weeks prior to entry; or use of traditional Chinese medicines for respiratory diseases. Patients provided written informed consent prior to any study-related procedure. Full inclusion and exclusion criteria are in the supplement.

After screening, eligible patients entered a two-week run-in receiving non-extrafine budesonide/formoterol (BUD/FF), 160/4.5 $\mu \mathrm{g}$, two puffs twice daily (BID) via dry-powder inhaler. They were then randomised equally to extrafine BDP/FF/G 100/6/10 $\mu$ g, two puffs BID via pressurised metered-dose inhaler, or to continue BUD/ FF for 24 weeks. Patients were assigned to treatment centrally via interactive response technology (IRT), using a balanced block randomisation scheme stratified by area that was generated by the IRT provider. Patients, investigators, and site and sponsor staff were blinded to treatment assignment by a double-dummy design, with matching placebos.

Patients attended visits at randomisation (baseline) and after 4, 12, 18 and 24 weeks. Lung function $\left[\mathrm{FEV}_{1}\right.$, forced vital capacity (FVC), forced mid-expiratory flow $\left(\mathrm{FEF}_{25-75 \%}\right)$, and inspiratory capacity (IC)] were assessed pre-dose at each visit, with $\mathrm{FEV}_{1}$ and $\mathrm{FVC}$ also assessed at 2-h post-dose. The COPD Assessment Test (CAT) was administered at each visit, with the St George's Respiratory Questionnaire (SGRQ) used at baseline and Weeks 12 and 24. COPD exacerbations were recorded throughout, with moderate exacerbations requiring treatment with systemic corticosteroids and/or antibiotics, and severe exacerbations resulting in hospitalisation or death. Salbutamol was permitted as rescue medication, with use recorded in diary cards. The occurrence of adverse events (AEs) was captured throughout, with safety evaluated by vital signs, electrocardiogram, haematology and blood chemistry.

The study was approved by independent ethics committees at each institution, and was performed in accordance with the principles of the Declaration of Helsinki, and the International Conference on Harmonisation notes for guidance on Good Clinical Practice 
(ICH/CPMP/135/95). Registered at the Chinese Food and Drug Administration (CFDA; registration number CTR20160507) and ClinicalTrials.gov (NCT03197818) websites.

\section{Outcomes}

The co-primary objectives were to demonstrate superiority of $\mathrm{BDP} / \mathrm{FF} / \mathrm{G}$ over BUD/FF in the overall population for change from baseline in pre-dose morning and 2-h post-dose $F E V_{1}$ at Week 24. To support regulatory approval of BDP/FF/G in China, the study's key secondary objectives were to assess the co-primary objectives in the subgroup of patients recruited at sites in China (the China subgroup). Other secondary endpoints included: change from baseline in pre-dose and 2-h post-dose $\mathrm{FEV}_{1}$ at other visits and overall; $\mathrm{FEV}_{1}$ response (change from baseline $\geq 100 \mathrm{~mL}$ ) at Week 24; change from baseline in pre-dose $\mathrm{FVC}, \mathrm{FEF}_{25-75 \%}$, and IC (a measure of static hyperinflation), and 2-h post-dose FVC at all visits and overall; time to first moderate-or-severe COPD exacerbation; rate of moderate/severe COPD exacerbations; change from baseline in SGRQ total score at Weeks 12 and 24 and overall, and in CAT at all visits and overall; SGRQ response (decrease from baseline $\geq 4$ ) at Week 24; change from baseline in rescue medication use in each inter-visit period and overall; and safety and tolerability.

\section{Sample size and statistical methods}

The study intended to randomise a total of 990 patients. An interim analysis was planned when 614 randomised patients completed the study [with an estimated 499 (81\%) in China]; assuming a non-evaluable rate of $13 \%$, 534 would be evaluable at Week 24, providing approximately $89 \%$ power ( $80 \%$ for the China subgroup) to detect mean differences in favour of BDP/FF/G of $60 \mathrm{~mL}$ for pre-dose morning $\mathrm{FEV}_{1}$ and $70 \mathrm{~mL}$ for post-dose $\mathrm{FEV}_{1}$, assuming standard deviations of $200 \mathrm{~mL}$ for both. To avoid inflation of the Type I error, significance was adjusted using the Pocock-type error spending function method (considering a two-sided alpha of 0.0372). For the interim analysis, an independent data monitoring committee (DMC) reviewed unblinded data, with the DMC charter specifying the study would be stopped if $\mathrm{BDP} / \mathrm{FF} / \mathrm{G}$ was superior to $\mathrm{BUD} / \mathrm{FF}$ for all co-primary and key secondary objectives. This was the case, and so further recruitment was stopped.

The co-primary endpoints were analysed using a linear mixed model for repeated measures including treatment, visit, treatment-by-visit interaction, area, COPD exacerbations in the previous year ( 1 or $>1)$, smoking status, and severity of airflow limitation (screening $\mathrm{FEV}_{1}<30 \%$ or $\geq 30 \%$ predicted) as fixed effects, and baseline value and baseline-by-visit interaction as covariates. The co-primary and key secondary endpoints were analysed in hierarchical order: (1) pre-dose and (2) post-dose $\mathrm{FEV}_{1}$ in the overall intention-to-treat (ITT) population; (3) pre-dose and (4) post-dose $\mathrm{FEV}_{1}$ in the China subgroup. No superiority claim was possible unless the preceding test was significant in favour of BDP/FF/G.

The changes from baseline in $\mathrm{FEV}_{1}, \mathrm{FVC}, \mathrm{FEF}_{25-75 \%}$, IC, SGRQ and CAT secondary endpoints were analysed using a similar model to the primary endpoints. A similar model was used to analyse the inter-visit rescue medication secondary endpoints, with inter-visit period used instead of visit in the model; the change from baseline for the entire treatment period was analysed using an analysis of covariance model including treatment, country, number of COPD exacerbations in the previous year and smoking status as fixed effects and the baseline value as a covariate. $\mathrm{FEV}_{1}$ response and SGRQ response were analysed using a logistic model including treatment, country, number of COPD exacerbations in the previous year and smoking status as factors and the baseline value as a covariate. The number of moderate/severe COPD exacerbations was analysed using a negative binomial model including treatment, country, number of COPD exacerbations in the previous year and smoking status as fixed effects, and log-time on study as an offset. The time to first COPD exacerbation was analysed using a Cox proportional hazards model including treatment, country, number of COPD exacerbations in the previous year and smoking status as factors.

Efficacy data were analysed in the ITT population, comprising all randomised patients who received at least one dose of study medication and with at least one postbaseline efficacy evaluation available. Safety analyses were performed in the safety population, which was all randomised patients who received at least one dose of study medication. The co-primary endpoints were also analysed in the per protocol (PP) population, which was all patients from the ITT population without any major protocol deviations. There was one protocol amendment after recruitment started, the main impact of which was to add the interim analysis. Data are presented overall and for the China subgroup.

\section{Results \\ Participants}

The study was conducted in 63 centres (China 41, Republic of Korea 14, Taiwan 8) between 14 December 2016 and 26 May 2020. Of 708 patients randomised, 88.8\% completed $(90.1 \%$ and $87.6 \%$ in the BDP/FF/G and BUD/ FF groups, respectively; Fig. 1). Disposition was similar for the China subgroup (Additional file 1: Figure S1). One patient in China did not receive study medication and was excluded from the BDP/FF/G safety and ITT 


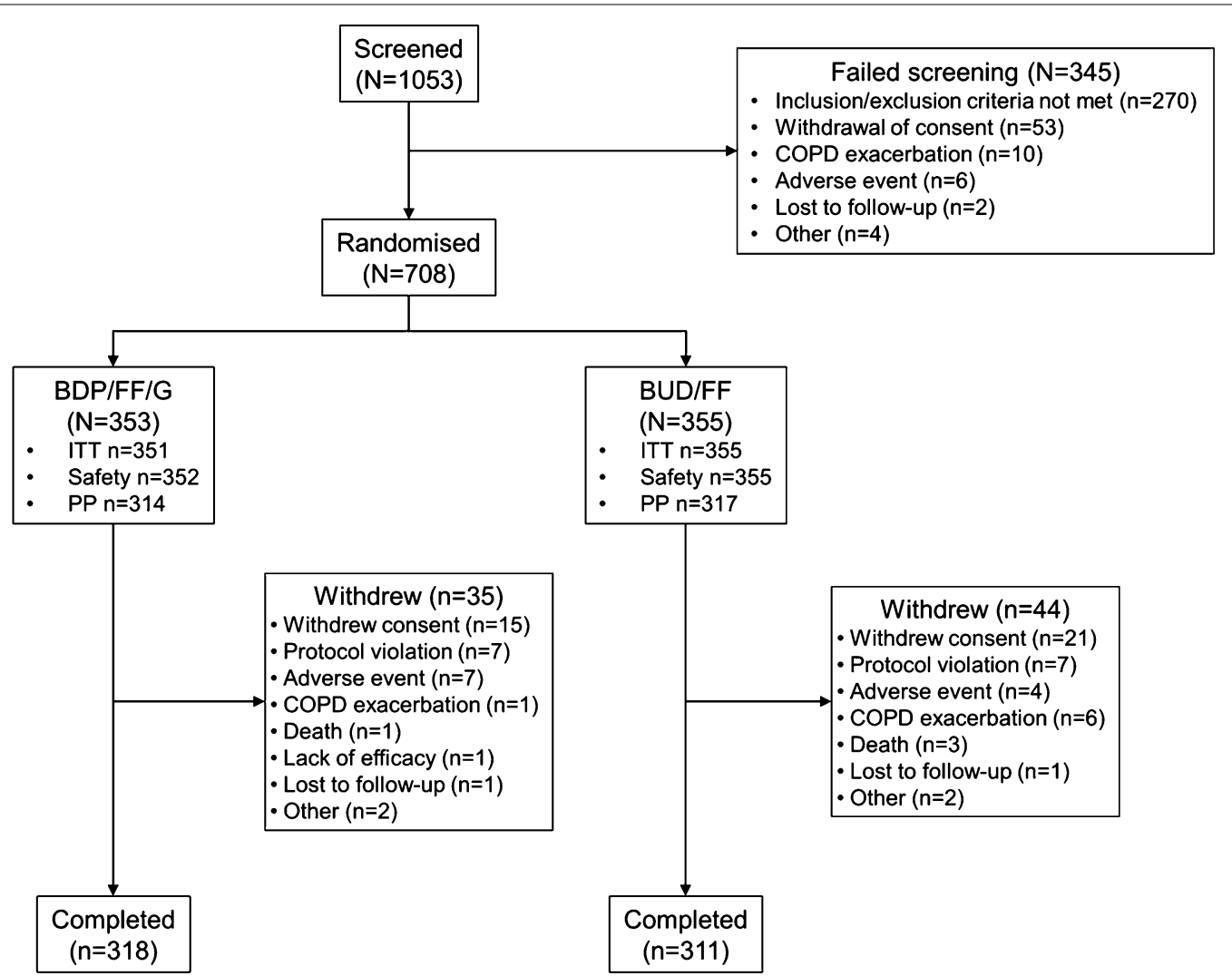

Fig. 1 Patient flow through the study (overall population). COPD chronic obstructive pulmonary disease, BDP beclometasone dipropionate, FF formoterol fumarate, G glycopyrronium, BUD budesonide, ITT intention-to-treat, PP per protocol

populations; another patient in China had no post-baseline efficacy data, and was excluded from the BDP/FF/G ITT population. Baseline characteristics were similar between treatment groups, overall and in the China subgroup (Table 1).

\section{Outcomes}

\section{Lung function}

In the overall population, for pre-dose and 2-h post-dose $\mathrm{FEV}_{1}$ at Week $24 \mathrm{BDP} / \mathrm{FF} / \mathrm{G}$ was superior to $\mathrm{BUD} / \mathrm{FF}$, with adjusted mean differences [95\% confidence interval (CI) ] of $62(38,85) \mathrm{mL}$ and $113(87,140) \mathrm{mL}$, respectively (both $p<0.001)$. Results were similar in the China subgroup $[63(36,90) \mathrm{mL}$ and $120(89,151) \mathrm{mL}$, respectively; $p<0.001]$. The results in the PP population were similar to those for the ITT population. Results at other visits were consistent with the Week 24 data, with significant $\mathrm{BDP} / \mathrm{FF} / \mathrm{G}$ versus BUD/FF differences in the overall population (Fig. 2a, b) and the China subgroup (Additional file 1: Figure S2).

At Week 24, 91 (25.9\%) patients had $\geq 100 \mathrm{~mL}$ change from baseline in pre-dose morning $\mathrm{FEV}_{1}$ in the BDP/ $\mathrm{FF} / \mathrm{G}$ group, compared with $43(12.1 \%)$ in the BUD/FF group, with a significant odds ratio [OR; 2.58 (95\% CI 1.72, 3.85); $p<0.001]$. Results were similar in the China subgroup [82 (28.7\%) and $39(13.4 \%)$ patients, respectively; OR 2.59 (1.70, 3.97); $p<0.001]$. FVC (pre-dose and 2-h post-dose) and $\mathrm{FEF}_{25-75 \%}$ (pre-dose) results were consistent with $\mathrm{FEV}_{1}$, with significant $\mathrm{BDP} / \mathrm{FF} / \mathrm{G}$ versus $\mathrm{BUD} / \mathrm{FF}$ differences at all visits in the overall population and the China subgroup (Additional file 1: Figures S3S5). There was a reduction in static lung hyperinflation in the BDP/FF/G group over the study (indicated by an increase from baseline in resting IC), with significant $\mathrm{BDP} / \mathrm{FF} / \mathrm{G}$ versus BUD/FF differences at all visits (Fig. 3; Additional file 1: Figure S6). This is important, as hyperinflation is one of the main reasons for activity limitation in patients with COPD, with reductions in hyperinflation associated with improvements in exercise tolerance [10-13].

\section{Exacerbations}

The annualised moderate/severe exacerbation rate was $43 \%$ lower overall with BDP/FF/G than BUD/FF, with a $50 \%$ reduction in the China subgroup $(p<0.001$; Fig. 4$)$. In the overall population, 66 (18.8\%) patients in the BDP/ 
Table 1 Patient baseline demographics and disease characteristics (ITT population)

\begin{tabular}{|c|c|c|c|c|}
\hline & \multicolumn{2}{|l|}{ Overall population } & \multicolumn{2}{|l|}{ China subgroup } \\
\hline & $\mathrm{BDP} / \mathrm{FF} / \mathrm{G}(\mathrm{N}=351)$ & $B U D / F F(N=355)$ & $\mathrm{BDP} / \mathrm{FF} / \mathrm{G}(\mathrm{N}=286)$ & $\mathrm{BUD} / \mathrm{FF}(\mathrm{N}=290)$ \\
\hline \multicolumn{5}{|l|}{ Areas, $n(\%)$} \\
\hline China & $286(81.5)$ & $290(81.7)$ & $286(100)$ & $290(100)$ \\
\hline Republic of Korea & $54(15.4)$ & $54(15.2)$ & - & - \\
\hline Taiwan & $11(3.1)$ & $11(3.1)$ & - & - \\
\hline Age, years & $66.0(7.1)$ & $65.9(7.7)$ & $65.3(7.1)$ & $65.3(7.4)$ \\
\hline Males, n (\%) & $336(95.7)$ & $337(94.9)$ & $271(94.8)$ & $273(94.1)$ \\
\hline Body-mass index, $\mathrm{kg} / \mathrm{m}^{2}$ & $21.8(3.5)$ & $21.3(3.3)$ & $21.8(3.4)$ & $21.5(3.4)$ \\
\hline \multicolumn{5}{|l|}{ Smoking status, n (\%) } \\
\hline Ex-smoker & $266(75.8)$ & $266(74.9)$ & $215(75.2)$ & $215(74.1)$ \\
\hline Current smoker & $85(24.2)$ & $89(25.1)$ & $71(24.8)$ & $75(25.9)$ \\
\hline Smoking history, pack-years & $39.9(24.0)$ & $41.3(22.5)$ & $39.0(23.9)$ & $40.8(23.0)$ \\
\hline Time since first COPD diagnosis (years) & $6.8(5.9)$ & $6.3(4.9)$ & $6.3(5.9)$ & $5.8(4.6)$ \\
\hline \multicolumn{5}{|l|}{ FEV ${ }_{1}$, post-bronchodilator } \\
\hline Percent predicted & $34.8(8.9)$ & $34.1(8.6)$ & $34.1(8.9)$ & $33.5(8.7)$ \\
\hline$<30 \%$ predicted, $\mathrm{n}(\%)$ & $113(32.2)$ & $119(33.5)$ & $103(36.0)$ & $106(36.6)$ \\
\hline $30-50 \%$ predicted, n (\%) & $238(67.8)$ & $236(66.5)$ & $183(64.0)$ & $184(63.4)$ \\
\hline Reversibility in $\mathrm{FEV}_{1}, \%$ & $12.7(12.7)$ & $12.5(12.4)$ & $13.8(13.2)$ & $13.0(12.7)$ \\
\hline CAT total score & $16.7(7.2)$ & $17.4(6.9)$ & $16.6(7.1)$ & $17.3(6.7)$ \\
\hline SGRQ total score & $44.1(18.2)$ & $43.3(17.3)$ & $43.9(17.3)$ & $43.1(16.7)$ \\
\hline Exacerbations in previous year, median (range) & $1.0(1,11)$ & $1.0(1,7)$ & $1.0(1,11)$ & $1.0(1,7)$ \\
\hline $1, \mathrm{n}(\%)$ & $253(72.1)$ & $261(73.5)$ & $218(76.2)$ & $226(77.9)$ \\
\hline $2, \mathrm{n}(\%)$ & $58(16.5)$ & $62(17.5)$ & $41(14.3)$ & $43(14.8)$ \\
\hline$\geq 3, \mathrm{n}(\%)$ & $40(11.4)$ & $32(9.0)$ & $27(9.4)$ & $21(7.2)$ \\
\hline \multicolumn{5}{|l|}{ COPD medication at study entry, $n(\%)$} \\
\hline ICS/LABA & $222(63.2)$ & $236(66.5)$ & $211(73.8)$ & $221(76.2)$ \\
\hline LABA/LAMA & $46(13.1)$ & $51(14.4)$ & $2(0.7)$ & $9(3.1)$ \\
\hline LAMA & $81(23.1)$ & $67(18.9)$ & $73(25.5)$ & $60(20.7)$ \\
\hline LABA & $2(0.6)$ & $1(0.3)$ & 0 & 0 \\
\hline
\end{tabular}

Data are mean (standard deviation) unless specified otherwise. ITT intention-to-treat, BDP beclometasone dipropionate, $F F$ formoterol fumarate, $G$ glycopyrronium, $B U D$ budesonide, COPD chronic obstructive pulmonary disease, FEV forced expiratory volume in $1 \mathrm{~s}$, CAT COPD assessment test, SGRQ St George's Respiratory Questionnaire, ICS inhaled corticosteroid, LABA long-acting beta2-agonist, LAMA long-acting muscarinic antagonist

FF/G group had $\geq 1$ moderate/severe COPD exacerbation, versus 109 (30.7\%) with BUD/FF; the time to first moderate-or-severe exacerbation significantly extended with $\mathrm{BDP} / \mathrm{FF} / \mathrm{G}$ [hazard ratio 0.55 (95\% CI 0.40, 0.75); $p<0.001$; Additional file 1: Figure S7A]. In the China subgroup, $49(17.1 \%)$ and $92(31.7 \%)$ patients, respectively, had an exacerbation, with the time to first moderate-orsevere COPD exacerbation again significantly longer in the BDP/FF/G group [0.48 (95\% CI 0.34, 0.68); $p<0.001$; Additional file 1: Figure S7B].

\section{Health status}

SGRQ and CAT total scores improved (scores decreased) from baseline with BDP/FF/G, but were unchanged with BUD/FF; differences between groups were significant in both SGRQ assessments, and at Weeks 12 and 24 (and also at Week 4 in the China subgroup) for the CAT assessments (Fig. 5; Additional file 1: Figure S8). At Week 24, 149 patients $(42.5 \%)$ overall were SGRQ responders ( $\geq 4$ unit decrease from baseline) with BDP/FF/G, compared to 119 (33.5\%) with BUD/FF [OR 1.48 (95\% CI 1.07, 2.05); $p=0.018$ ]. In the China subgroup, 127 (44.4\%) and $97(33.4 \%)$ patients, respectively, were responders [1.61 $(1.12,2.30 ; p=0.010)]$.

\section{Rescue medication use}

Rescue medication use decreased from baseline in both groups. The decrease with BDP/FF/G was larger, although differences between groups were not consistently significant (Additional File 1: Figures S9 and S10). 


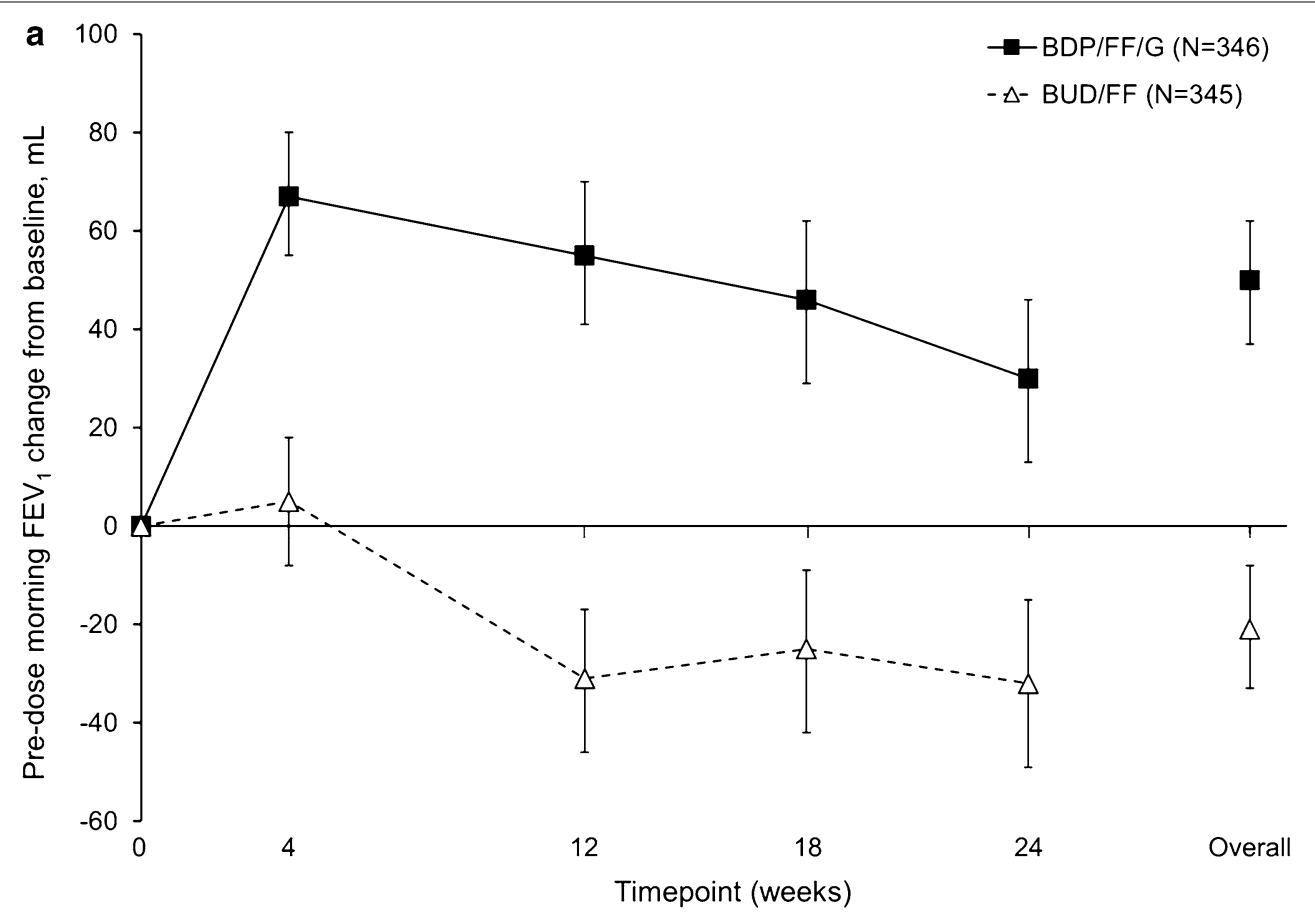

BDP/FF/G vs BUD/FF adjusted mean difference, $\mathrm{mL}(95 \% \mathrm{Cl})$
$62(44,80)^{\ddagger}$
$87(66,107)^{\ddagger}$
$71(48,94)^{\ddagger}$
$62(38,85)^{\ddagger}$
$70(53,88)^{\ddagger}$

b
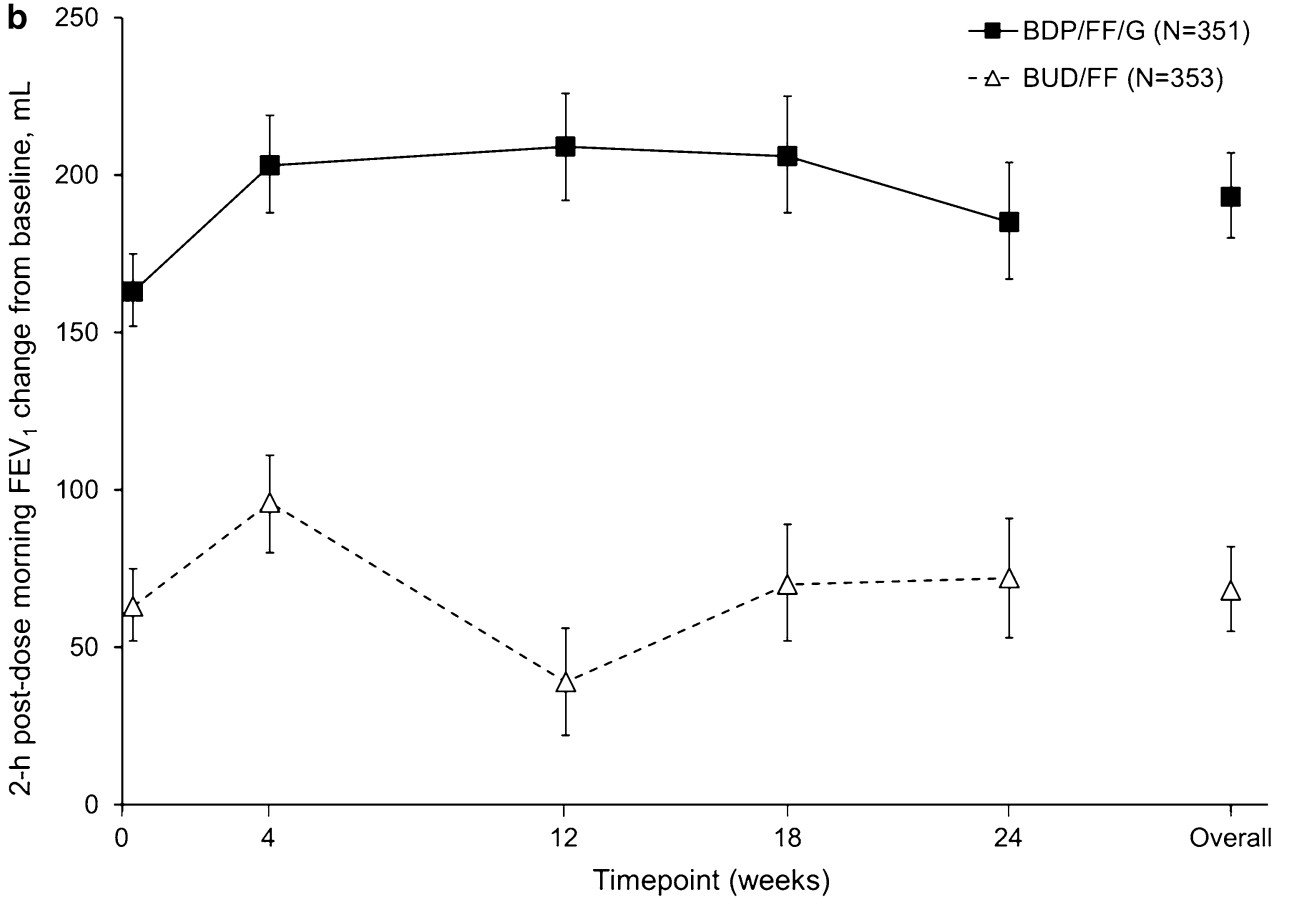

BDP/FF/G vs BUD/FF adjusted mean difference, $\mathrm{mL}(95 \% \mathrm{Cl})$

$100(83,117)^{\ddagger} \quad 107(85,129)^{\ddagger} \quad 170(146,194)^{\ddagger} \quad 136(110,162)^{\ddagger} \quad 113(87,140)^{\ddagger} \quad 125(106,144)^{\ddagger}$

Fig. 2 Adjusted mean change from baseline in a pre-dose morning $\mathrm{FEV}_{1}$, and $\mathbf{b} 2$-h post-dose $\mathrm{FEV}_{1}$ (overall population, ITT). ${ }^{\ddagger} p<0.001$. Data are adjusted mean and $95 \% \mathrm{Cl}$. The $\mathrm{N}$ values are the number of patients included in the statistical model. FEV forced expiratory volume in $1 \mathrm{~s}$, ITT intention-to-treat, BDP beclometasone dipropionate, FF formoterol fumarate, $\mathrm{G}$ glycopyrronium, BUD budesonide, $C /$ confidence interval 


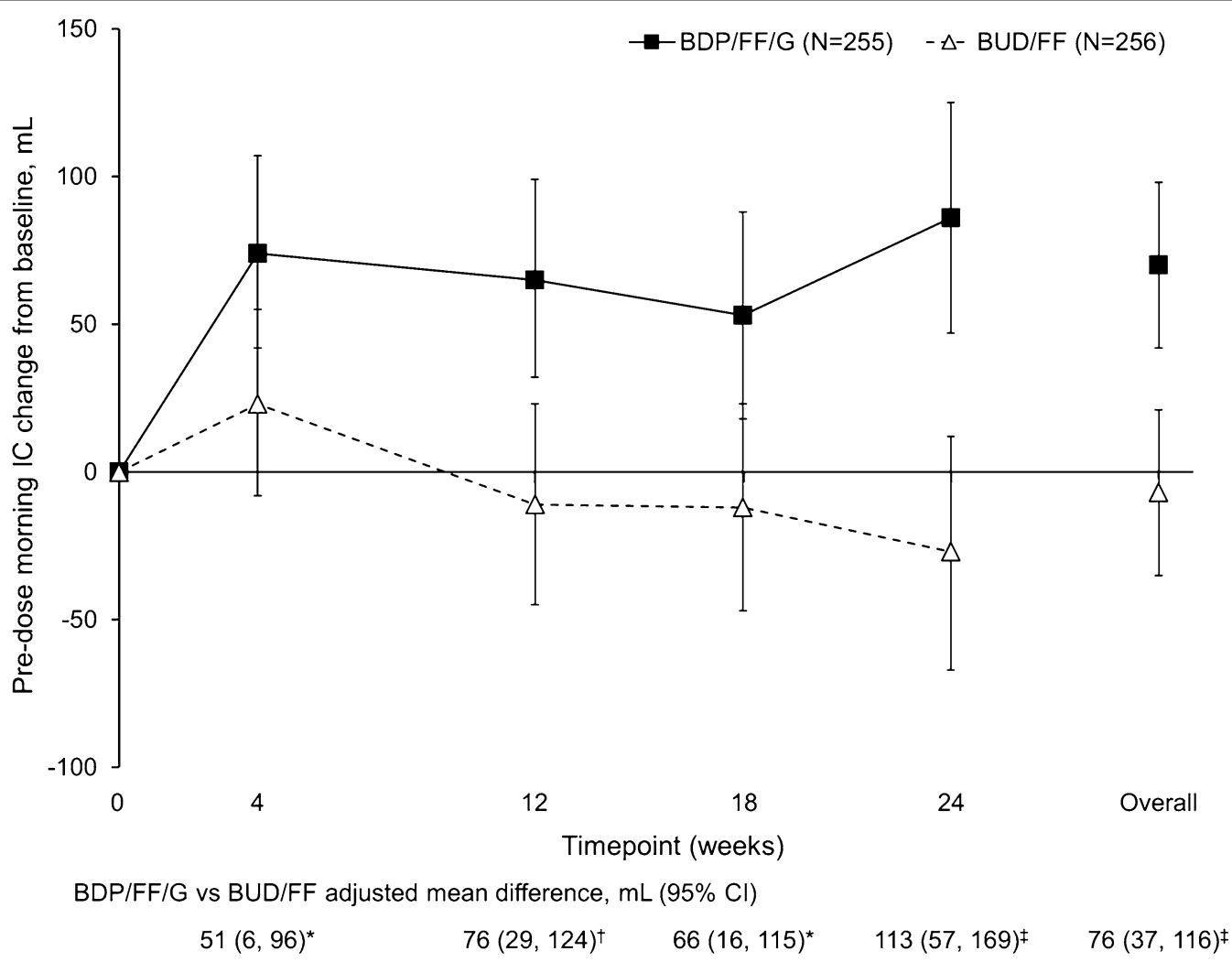

Fig. 3 Adjusted mean change from baseline in IC (overall population, ITT). ${ }^{*} p<0.05 ;{ }^{\dagger} p<0.01 ;{ }^{\ddagger} p<0.001$. Data are adjusted mean and $95 \% \mathrm{Cl}$. The N values are the number of patients included in the statistical model. IC inspiratory capacity, ITT intention-to-treat, BDP beclometasone dipropionate, FF formoterol fumarate, G glycopyrronium, BUD budesonide, Cl confidence interval

\section{Safety}

Fewer patients experienced AEs with BDP/FF/G than BUD/FF, predominantly due to fewer COPD exacerbations (Table 2). The majority of AEs were mild or moderate, with few study-related. The only treatmentrelated serious $\mathrm{AE}$ was liver injury, in a patient in the China subgroup receiving $\mathrm{BDP} / \mathrm{FF} / \mathrm{G}$; this patient subsequently recovered. Four patients died during the study, all in the China subgroup, none studyrelated. One patient was receiving $\mathrm{BDP} / \mathrm{FF} / \mathrm{G}$ (the $\mathrm{AE}$ was recorded only as death). The only AE leading to study-drug discontinuation in more than one patient with either treatment was COPD exacerbation: One patient with BDP/FF/G and seven with BUD/FF. Fewer patients had pneumonia AEs with $\mathrm{BDP} / \mathrm{FF} / \mathrm{G}$ than BUD/FF, both overall (2.3\% versus $3.7 \%)$ and in the China subgroup (1.4\% versus $2.4 \%$ ).

There were few clinically relevant changes in haematology or biochemistry parameters, and minimal changes in any vital sign parameters. The incidence of clinically relevant QTc interval values was low and not different between treatments; no males had QTc interval values $>480 \mathrm{~ms}$, and no females had values $>500 \mathrm{~ms}$, and there were no changes $>60 \mathrm{~ms}$.

\section{Discussion}

In this study, triple therapy with extrafine BDP/FF/G improved lung function and health status compared with non-extrafine BUD/FF, with significant reductions in the incidence and rate of COPD exacerbations. The results in the China subgroup were consistent with the overall population, with BDP/FF/G demonstrating similar efficacy to the overall population and a good overall safety profile.

Our results are consistent with those of a number of previous 52-week triple therapy versus ICS/LABA studies $[6,14-16]$, although we specifically recruited only patients from eastern Asia. In particular, the results of the TRILOGY study, in which differences at Week 26 between BDP/FF/G and BDP/FF were 81 and $117 \mathrm{~mL}$ for pre-dose and 2-h post-dose $\mathrm{FEV}_{1}$, respectively [6], were similar to those observed here $(62 \mathrm{~mL}$ and $113 \mathrm{~mL}$, respectively). The TRIVERSYTI study design specified 


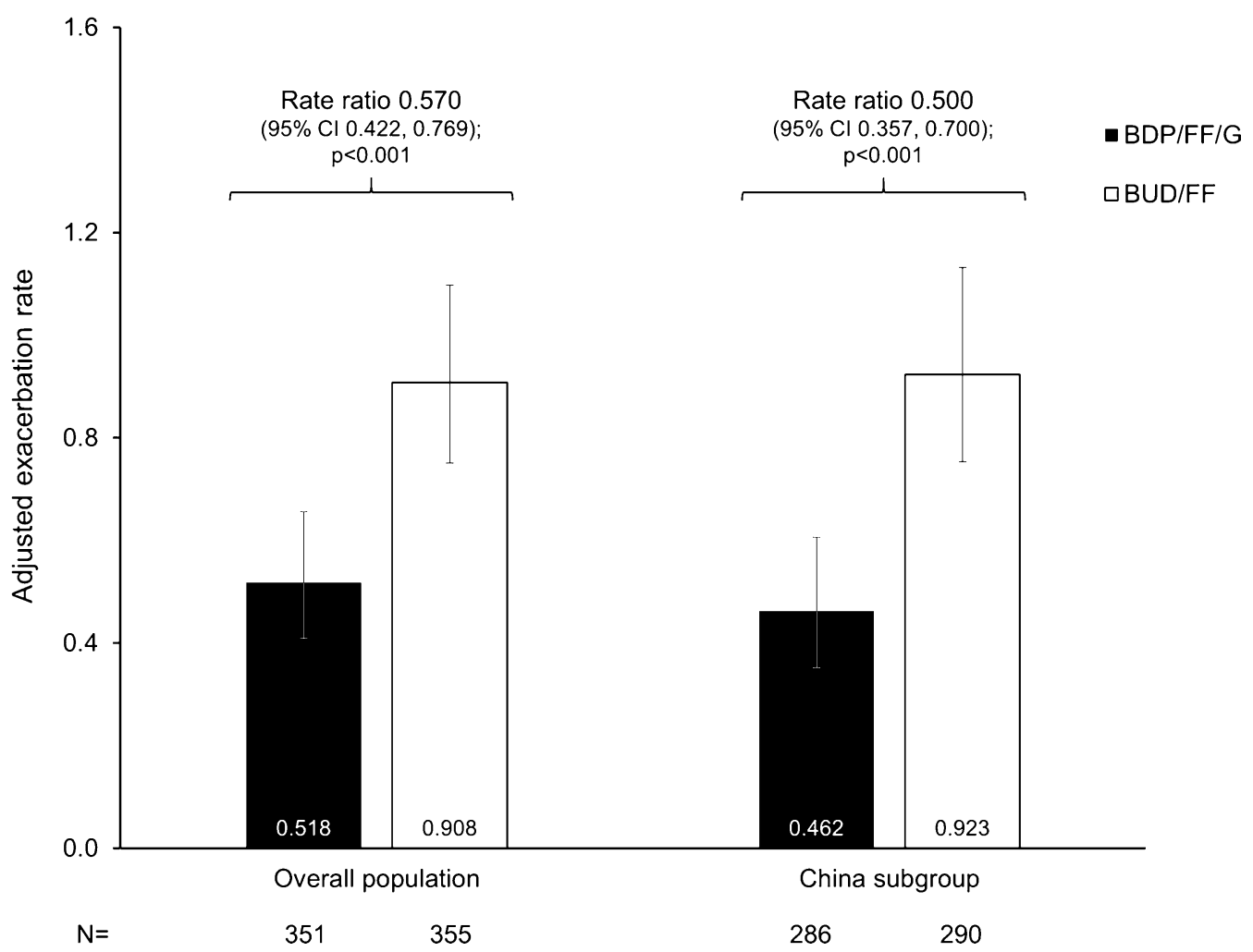

Fig. 4 Annualised moderate/severe COPD exacerbation rates (ITT population). Bars represent adjusted exacerbation rate per patient per year and $95 \% \mathrm{CI}$. The $\mathrm{N}$ values are for the ITT population. COPD chronic obstructive pulmonary disease, ITT intention-to-treat, BDP beclometasone dipropionate, FF formoterol fumarate, G glycopyrronium, BUD budesonide, $\mathrm{Cl}$ confidence interval

that recruitment should be stopped after 614 randomised patients were evaluated if statistically significant outcomes were observed for the co-primary and key secondary objectives. Although there would be no overt harm in continuing to recruit patients into the study, the effect of BDP/FF/G on these objectives was sufficiently marked (and positive) that the independent DMC recommended that no further subject recruitment into TRIVERSYTI was necessary.

While the TRIVERSYTI study primary analysis was focused on lung function endpoints, the enrolled patients were required to have had at least one exacerbation in the previous year, to recruit patients at increased exacerbation risk, such that treatment differences on exacerbation rate could be properly evaluated. The annualised moderate/severe exacerbation rate in the ICS/LABA group in TRIVERSYTI (0.91) was higher than in TRILOGY (0.56), as was the rate reduction ( $43 \%$ versus $23 \%)$. The large effect size for such a modest sample size suggests that the population recruited into TRIVERSYTI may gain even more benefit from treatment-although the ICS/LABAs differed in the two studies, with extrafine BDP/FF used in TRILOGY and non-extrafine BUD/FF in TRIVERSYTI. Importantly, the improved efficacy of $\mathrm{BDP} / \mathrm{FF} / \mathrm{G}$ versus BUD/FF was accompanied by an overall good safety profile-and in particular with a lower incidence of pneumonia. This lower pneumonia incidence could be due to differences in the ICS, the dose or formulation (extrafine $\mathrm{BDP}$, compared to non-extrafine BUD); however, given the addition of the LAMA to ICS/LABA reduces the incidence of exacerbations, one may expect this reduced pneumonia risk from BDP/FF/G.

To our knowledge, this is the first study specifically designed to evaluate the efficacy of single-inhaler triple

(See figure on next page.)

Fig. 5 Adjusted mean change from baseline in a SGRQ and $\mathbf{b}$ CAT total scores (overall population, ITT). ${ }^{\dagger} p<0.01 ;{ }^{*} p<0.001$. Data are adjusted mean and $95 \% \mathrm{Cl}$. The N values are the number of patients included in the statistical model. SGRQ St George's Respiratory Questionnaire, CAT COPD Assessment Test, ITT intention-to-treat, BDP beclometasone dipropionate, FF formoterol fumarate, G glycopyrronium, BUD budesonide, CI confidence interval 


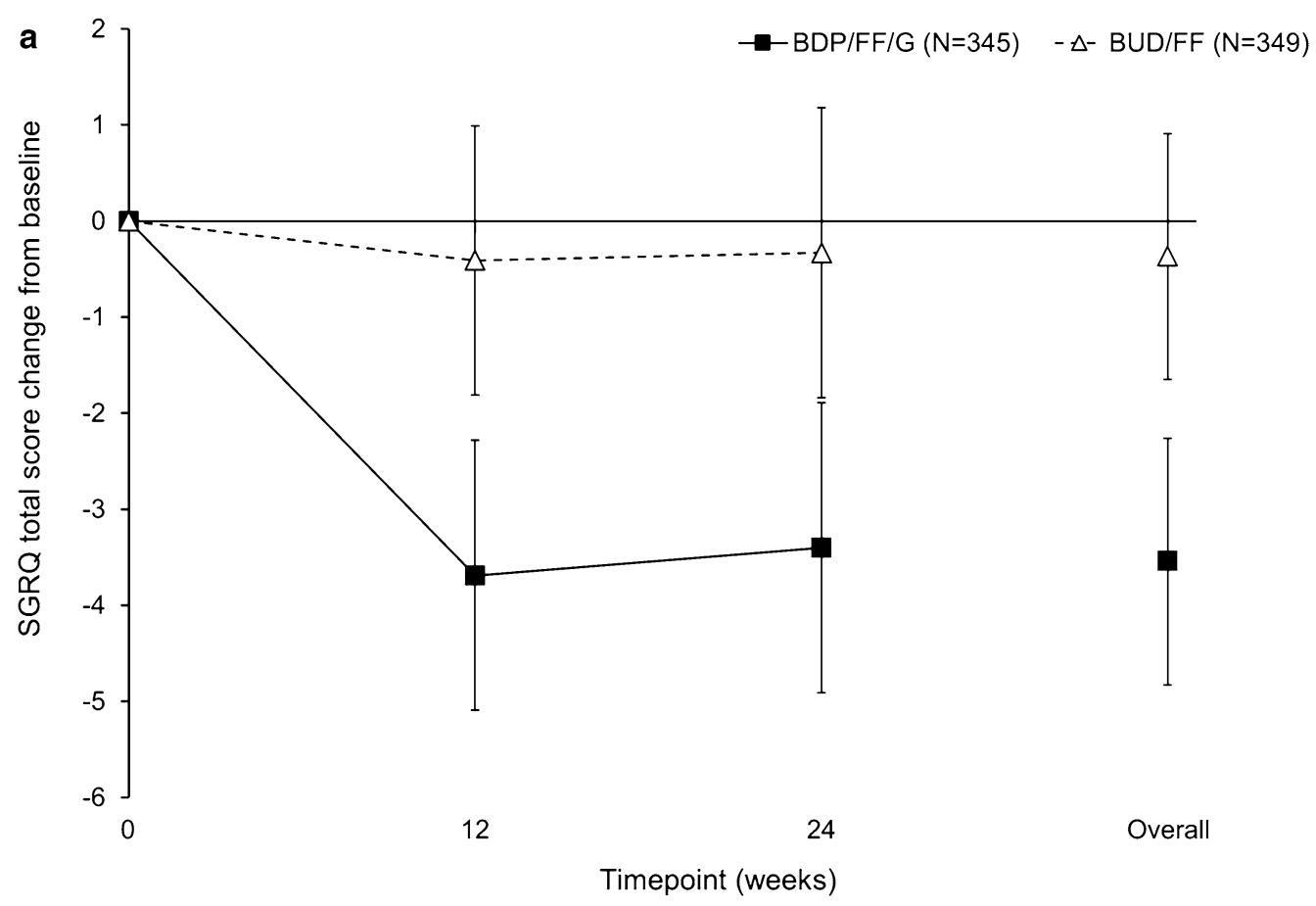

BDP/FF/G vs BUD/FF adjusted mean difference $(95 \% \mathrm{Cl})$

$$
-3.28(-5.26,-1.30)^{\dagger} \quad-3.07(-5.21,-0.94)^{\dagger} \quad-3.18(-4.99,-1.37)^{\ddagger}
$$

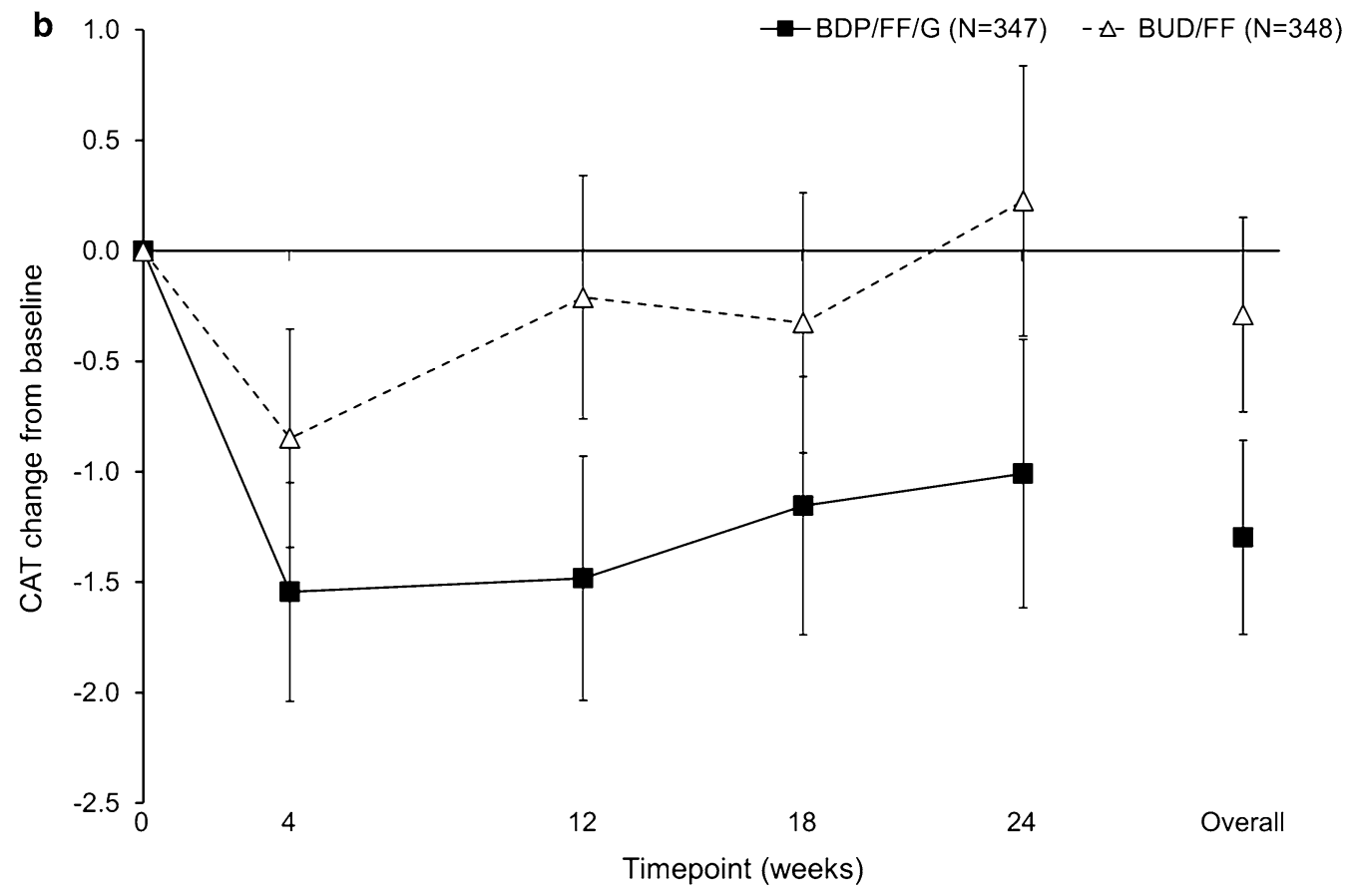

$\mathrm{BDP} / \mathrm{FF} / \mathrm{G}$ vs BUD/FF adjusted mean difference $(95 \% \mathrm{Cl})$

$\begin{array}{ccccc}-0.70 & -1.27^{\dagger} & -0.83 & -1.23^{\dagger} & -1.01^{\dagger} \\ (-1.40,0.00) & (-2.05,-0.49) & (-1.66,0.00) & (-2.10,-0.37) & (-1.63,-0.39)\end{array}$


Table 2 Adverse events and serious adverse events (safety population)

\begin{tabular}{|c|c|c|c|c|}
\hline \multirow[t]{2}{*}{ Number (\%) of patients } & \multicolumn{2}{|l|}{ Overall population } & \multicolumn{2}{|l|}{ China subgroup } \\
\hline & $\mathrm{BDP} / \mathrm{FF} / \mathrm{G}(\mathrm{N}=352)$ & $\mathrm{BUD} / \mathrm{FF}(\mathrm{N}=355)$ & $\mathrm{BDP} / \mathrm{FF} / \mathrm{G}(\mathrm{N}=287)$ & $\mathrm{BUD} / \mathrm{FF}(\mathrm{N}=290)$ \\
\hline Adverse events & $215(61.1)$ & $238(67.0)$ & $173(60.3)$ & $195(67.2)$ \\
\hline COPD exacerbation & $66(18.8)$ & $110(31.0)$ & $49(17.1)$ & $93(32.1)$ \\
\hline Upper respiratory tract infection & $58(16.5)$ & $49(13.8)$ & $55(19.2)$ & $45(15.5)$ \\
\hline Nasopharyngitis & $22(6.3)$ & $23(6.5)$ & $16(5.6)$ & $17(5.9)$ \\
\hline Hypertension & $12(3.4)$ & $24(6.8)$ & $10(3.5)$ & $19(6.6)$ \\
\hline Productive cough & $9(2.6)$ & $9(2.5)$ & $8(2.8)$ & $8(2.8)$ \\
\hline Pneumonia & $8(2.3)$ & $13(3.7)$ & $4(1.4)$ & $7(2.4)$ \\
\hline Cough & $8(2.3)$ & $8(2.3)$ & $4(1.4)$ & $7(2.4)$ \\
\hline Bronchitis & $6(1.7)$ & $3(0.8)$ & $5(1.7)$ & $1(0.3)$ \\
\hline Pharyngitis & $5(1.4)$ & $3(0.8)$ & $5(1.7)$ & $3(1.0)$ \\
\hline Dyspnoea & $4(1.1)$ & $8(2.3)$ & $3(1.0)$ & $5(1.7)$ \\
\hline Gamma-glutamyltransferase increased & $2(0.6)$ & $6(1.7)$ & $2(0.7)$ & $4(1.4)$ \\
\hline Hepatic function abnormal & $2(0.6)$ & $6(1.7)$ & $2(0.7)$ & $6(2.1)$ \\
\hline Alanine aminotransferase increased & $2(0.6)$ & $6(1.7)$ & $1(0.3)$ & $3(1.0)$ \\
\hline Back pain & 0 & $8(2.3)$ & 0 & $8(2.8)$ \\
\hline Hypokalaemia & 0 & $7(2.0)$ & 0 & $5(1.7)$ \\
\hline Neutrophil count increased & $6(1.7)$ & $2(0.6)$ & $6(2.1)$ & $1(0.3)$ \\
\hline Anaemia & $4(1.1)$ & $5(1.4)$ & $4(1.4)$ & $5(1.7)$ \\
\hline Lung infection & $2(0.6)$ & $5(1.4)$ & $2(0.7)$ & $5(1.7)$ \\
\hline Blood pressure increased & $1(0.3)$ & $5(1.4)$ & $1(0.3)$ & $5(1.7)$ \\
\hline Serious adverse events & $40(11.4)$ & $60(16.9)$ & $30(10.5)$ & $49(16.9)$ \\
\hline COPD exacerbation & $20(5.7)$ & $43(12.1)$ & $16(5.6)$ & $38(13.1)$ \\
\hline Pneumonia & $4(1.1)$ & $9(2.5)$ & $1(0.3)$ & $4(1.4)$ \\
\hline Pneumothorax spontaneous & $2(0.6)$ & 0 & $2(0.7)$ & 0 \\
\hline Influenza & $2(0.6)$ & 0 & 0 & 0 \\
\hline Treatment-related adverse events & $9(2.6)$ & $16(4.5)$ & $8(2.8)$ & $14(4.8)$ \\
\hline Muscle spasms & $2(0.6)$ & $2(0.6)$ & $2(0.7)$ & $2(0.7)$ \\
\hline Blood pressure increased & 0 & $2(0.6)$ & 0 & $2(0.7)$ \\
\hline Treatment-related serious adverse events & $1(0.3)$ & 0 & $1(0.3)$ & 0 \\
\hline Severe adverse events & $31(8.8)$ & $56(15.8)$ & $23(8.0)$ & $48(16.6)$ \\
\hline COPD exacerbation & $22(6.3)$ & $46(13.0)$ & $18(6.3)$ & $41(14.1)$ \\
\hline Pneumonia & $3(0.9)$ & $8(2.3)$ & $2(0.7)$ & $4(1.4)$ \\
\hline Influenza & $2(0.6)$ & 0 & 0 & 0 \\
\hline Lung infection & $1(0.3)$ & $2(0.6)$ & $1(0.3)$ & $2(0.7)$ \\
\hline Adverse events leading to study drug discontinuation & $8(2.3)$ & $13(3.7)$ & $8(2.8)$ & $10(3.4)$ \\
\hline COPD exacerbation & $1(0.3)$ & $7(2.0)$ & $1(0.3)$ & $5(1.7)$ \\
\hline Adverse events leading to death & $1(0.3)$ & $3(0.8)$ & $1(0.3)$ & $3(1.0)$ \\
\hline
\end{tabular}

Data are $\mathrm{n}(\%) . \geq 1.5 \%$ in either group of the overall population for adverse events and $\geq 0.5 \%$ in either group of the overall population for serious adverse events, treatment-related adverse events, and adverse events leading to study drug discontinuation. $B D P$ beclometasone dipropionate, $F F$ formoterol fumarate, $G$ glycopyrronium, BUD budesonide, COPD chronic obstructive pulmonary disease

therapy in patients from eastern Asia. One previous study evaluated the efficacy of tiotropium plus BUD/FF in five eastern Asian areas, in which triple therapy improved bronchodilation and health status compared with tiotropium alone, with a $40.7 \%$ reduction in exacerbation rate (of note, patients in the tiotropium group were not permitted ICS) [17]. Subgroup analyses in patients from
China have been published from two other single-inhaler triple therapy studies: in FULFIL, triple therapy with fluticasone furoate/umeclidinium/vilanterol improved bronchodilation compared with BUD/FF, with numerical improvements in health status and exacerbation rate [18], whereas in KRONOS BUD/glycopyrrolate/FF improved bronchodilation and health status versus BUD/FF, with 
a non-statistically significant $49 \%$ reduction in moderate/severe exacerbation rate [19]. Our results are broadly consistent with these, but with a much larger sample size, and with a statistically significant reduction in the rate of moderate/severe exacerbations.

We recognise that TRIVERSYTI has some limitations. Unlike in TRILOGY, the comparator in TRIVERSYTI differed in terms of molecules, formulation and device (when TRIVERSYTI was initiated, BDP/FF was not approved for COPD in China, Republic of Korea or Taiwan). It is unclear, therefore, which characteristic (or characteristics) was responsible for the observed improved efficacy (although the inclusion of the LAMA component in BDP/FF/G is likely responsible for much of the difference, as we observed in TRILOGY [6]). This reflects standard clinical practice, however, in that treatment choices are based not only on molecules but also on inhaler devices. In addition, more than $80 \%$ of patients were recruited by sites in China, and so the results of the overall population are largely driven by the China subgroup.

\section{Conclusions}

In conclusion, this study is the first to evaluate singleinhaler triple therapy in patients recruited solely in eastern Asia. In patients with COPD, $\mathrm{FEV}_{1}<50 \%$ and an exacerbation history despite maintenance therapy, treatment with extrafine BDP/FF/G improved bronchodilation and health status, and was more effective at preventing moderate/severe COPD exacerbations than $\mathrm{BUD} / \mathrm{FF}$.

\begin{abstract}
Abbreviations
AE: Adverse event; BDP: Beclometasone dipropionate; BID: Twice daily; BUD: Budesonide; CAT: COPD Assessment Test; Cl: Confidence interval; COPD: Chronic obstructive pulmonary disease; DMC: Data monitoring committee; $\mathrm{FEF}_{25-75 \%}$ : Forced mid-expiratory flow; $\mathrm{FEV}_{1}$ : Forced expiratory volume in $1 \mathrm{~s}$; FF: Formoterol fumarate; FVC: Forced vital capacity; G: Glycopyrronium; IC: Inspiratory capacity; ICS: Inhaled corticosteroid; IRT: Interactive response technology; ITT: Intention-to-treat; LABA: Long-acting $\beta_{2}$-agonist; LAMA: Long-acting muscarinic antagonist; OR: Odds ratio; PP: Per protocol; SGRQ: St George's Respiratory Questionnaire.
\end{abstract}

\section{Supplementary Information}

The online version contains supplementary material available at https://doi. org/10.1186/s12931-021-01683-2.

Additional file 1. Supplementary methods and results supporting the main body of the manuscript.

\section{Acknowledgements}

Results of this study have been presented at the American Thoracic Society 2020 annual conference. Dave Singh is supported by the National Institute for Health Research (NIHR) Manchester Biomedical Research Centre (BRC). The authors would like to thank the investigators and patients at the investigative sites for their support of this study.

\section{Authors' contributions}

The study was conceived and designed by SB, DS, AP, AG and GG. SB and FG oversaw conduct of the study. The data were acquired by JZ, LZ, HL and KHL, analysed by $A G$, and interpreted by all authors. The manuscript was revised for intellectual content by all authors. All authors read and approved the final manuscript.

\section{Funding}

This study was funded by Chiesi Farmaceutici SpA. Writing support was provided by David Young of Young Medical Communications and Consulting Ltd. This support was funded by Chiesi Farmaceutici SpA.

\section{Availability of data and materials}

Chiesi commits to sharing with qualified scientific and medical Researchers, conducting legitimate research, patient-level data, study-level data, the clinical protocol and the full clinical study report of Chiesi Farmaceutici SpA-sponsored interventional clinical trials in patients for medicines and indications approved by the European Medicines Agency and/or the US Food and Drug Administration after 1st January 2015, following the approval of any received research proposal and the signature of a Data Sharing Agreement. Chiesi provides access to clinical trial information consistently with the principle of safeguarding commercially confidential information and patient privacy. Other information on Chiesi's data sharing commitment, access and research request's approval process are available in the Clinical Trial Transparency section of http://www.chiesi.com/en/research-and-development/.

\section{Declarations}

Ethics approval and consent to participate

The study was approved by the independent ethics committees at each institution. All patients provided written informed consent prior to any studyrelated procedure.

\section{Consent for publication}

Not applicable.

\section{Competing interests}

JZ has no relevant conflicts to disclose. LZ has no relevant conflicts to disclose. HL has no relevant conflicts to disclose. K-HL has no relevant conflicts to disclose. DS reports personal fees from Chiesi during the conduct of the study. Outside the submitted work, he reports personal fees from AstraZeneca, Boehringer Ingelheim, Chiesi, Cipla, Genentech, GlaxoSmithKline, Glenmark, Menarini, Mundipharma, Novartis, Peptinnovate, Pfizer, Pulmatrix, Theravance, and Verona. AP reports grants, personal fees, non-financial support and payment for advisory board membership, consultancy, payment for lectures, grants for research, and travel expenses reimbursement from Chiesi, AstraZeneca, GlaxoSmithKline, Boehringer Ingelheim, Mundipharma and TEVA, and personal fees and non-financial support from Menarini, Novartis, Zambon and Sanofi, all outside the submitted work. SB, FG, AG and GG are employees of Chiesi, the sponsor of the study.

\section{Author details}

1 State Key Laboratory of Respiratory Disease, National Clinical Research Centre for Respiratory Disease, Guangzhou Institute of Respiratory Health, First Affiliated Hospital of Guangzhou Medical University, Guangzhou, China. ${ }^{2}$ Global Clinical Development, Chiesi Farmaceutici SpA, Largo Belloli, $11 \backslash a$, 43122 Parma, Italy. ${ }^{3}$ Shengjing Hospital of China Medical University, Shenyang, China. ${ }^{4}$ Shanghai Pulmonary Hospital, Shanghai, China. ${ }^{5}$ Yeungnam University Medical Center, Daegu, Republic of Korea. ${ }^{6}$ Medicines Evaluation Unit, The University of Manchester, Manchester University NHS Foundations Trust, Manchester, UK. ${ }^{7}$ Respiratory Medicine Unit, University of Ferrara, University Hospital S. Anna, Ferrara, Italy.

Received: 26 January 2021 Accepted: 11 March 2021 Published online: 23 March 2021 


\section{References}

1. Global Initiative for Chronic Obstructive Lung Disease. Global strategy for the diagnosis, management, and prevention of chronic obstructive pulmonary disease [Internet]. 2021 [cited 2020 Nov 25]. https://goldcopd. org/2021-gold-reports/.

2. Braido F, Scichilone N, Lavorini F, Usmani OS, Dubuske L, Boulet LP, et al. Manifesto on small airway involvement and management in asthma and chronic obstructive pulmonary disease: an Interasma (Global Asthma Association - GAA) and World Allergy Organization (WAO) document. World Allergy Organ J. 2016;9:1-6. https://doi.org/10.1186/ s40413-016-0123-2.

3. Higham A, Quinn AM, Cançado JED, Singh D. The pathology of small airways disease in COPD: historical aspects and future directions. Respir Res. 2019;20:49. https://doi.org/10.1186/s12931-019-1017-y.

4. Koo H-K, Vasilescu DM, Booth S, Hsieh A, Katsamenis OL, Fishbane N, et al. Small airways disease in mild and moderate chronic obstructive pulmonary disease: a cross-sectional study. Lancet Respir Med. 2018;6:591-602. https://doi.org/10.1016/S2213-2600(18)30196-6.

5. Usmani OS, Biddiscombe MF, Barnes PJ. Regional lung deposition and bronchodilator response as a function of beta2-agonist particle size. Am J Respir Crit Care Med. 2005;172:1497-504. https://doi.org/10.1164/rccm. 200410-14140C.

6. Singh D, Papi A, Corradi M, Pavlišová I, Montagna I, Francisco C, et al. Single inhaler triple therapy versus inhaled corticosteroid plus long-acting ß2-agonist therapy for chronic obstructive pulmonary disease (TRILOGY): a double-blind, parallel group, randomised controlled trial. Lancet. 2016;388:963-73. https://doi.org/10.1016/S0140-6736(16)31354-X.

7. Vestbo J, Papi A, Corradi M, Blazhko V, Montagna I, Francisco C, et al. Single inhaler extrafine triple therapy versus long-acting muscarinic antagonist therapy for chronic obstructive pulmonary disease (TRINITY): a double-blind, parallel group, randomised controlled trial. Lancet. 2017;389:1919-29. https://doi.org/10.1016/S0140-6736(17)30188-5.

8. Papi A, Vestbo J, Fabbri L, Corradi M, Prunier H, Cohuet G, et al. Extrafine inhaled triple therapy versus dual bronchodilator therapy in chronic obstructive pulmonary disease (TRIBUTE): a double-blind, parallel group, randomised controlled trial. Lancet. 2018;391:1076-84. https://doi.org/10. 1016/S0140-6736(18)30206-X

9. Fang L, Gao P, Bao H, Tang X, Wang B, Feng Y, et al. Chronic obstructive pulmonary disease in China: a nationwide prevalence study. Lancet Respir Med. 2018;6:421-30. https://doi.org/10.1016/S2213-2600(18) 30103-6.

10. Ofir D, Laveneziana P, Webb KA, Lam Y-M, O'Donnell DE. Mechanisms of dyspnea during cycle exercise in symptomatic patients with GOLD stage I chronic obstructive pulmonary disease. Am J Respir Crit Care Med. 2008;177:622-9.
11. Beeh K-M, Wagner F, Khindri S, Drollmann AF. Effect of indacaterol on dynamic lung hyperinflation and breathlessness in hyperinflated patients with COPD. COPD. 2011;8:340-5.

12. O'Donnell DE, Casaburi R, Vincken W, Puente-Maestu L, Swales J, Lawrence $D$, et al. Effect of indacaterol on exercise endurance and lung hyperinflation in COPD. Respir Med. 2011;105:1030-6.

13. Watz H, Mailänder C, Baier M, Kirsten A. Effects of indacaterol/glycopyrronium (QVA149) on lung hyperinflation and physical activity in patients with moderate to severe COPD: a randomised, placebo-controlled, crossover study (The MOVE Study). BMC Pulm Med. 2016;16:95. https:// doi.org/10.1186/s12890-016-0256-7.

14. Lipson DA, Barnhart F, Brealey N, Brooks J, Criner GJ, Day NC, et al. Oncedaily single-inhaler triple versus dual therapy in patients with COPD. N Engl J Med. 2018;378:1671-80. https://doi.org/10.1056/NEJMoa1713901.

15. Lipson DA, Barnacle H, Birk R, Brealey N, Locantore N, Lomas DA, et al. FULFIL Trial: once-daily triple therapy for patients with chronic obstructive pulmonary disease. Am J Respir Crit Care Med. 2017;196:438-46. https://doi.org/10.1164/rccm.201703-04490C.

16. Rabe KF, Martinez FJ, Ferguson GT, Wang C, Singh D, Wedzicha JA, et al. Triple inhaled therapy at two glucocorticoid doses in moderate-to-verysevere COPD. N Engl J Med. 2020;383:35-48. https://doi.org/10.1056/ NEJMoa1916046.

17. Lee S-D, Xie C-MM, Yunus F, Itoh Y, Ling X, Yu WC, et al. Efficacy and tolerability of budesonide/formoterol added to tiotropium compared with tiotropium alone in patients with severe or very severe COPD: A randomized, multicentre study in East Asia. Respirology. 2016;21:119-27. https://doi.org/10.1111/resp.12646.

18. Zheng J, Zhong N, Wang C, Huang Y, Chen P, Wang L, et al. The efficacy and safety of once-daily fluticasone furoate/umeclidinium/vilanterol versus twice-daily budesonide/formoterol in a subgroup of patients from China with symptomatic COPD at risk of exacerbations (FULFIL Trial). COPD J Chronic Obstr Pulm Dis. 2018;15:334-40. https://doi.org/10.1080/ 15412555.2018 .1481022$.

19. Wang C, Yang T, Kang J, Chen R, Zhao L, He H, et al. Efficacy and safety of budesonide/glycopyrrolate/formoterol fumarate metered dose inhaler in Chinese patients with COPD: a subgroup analysis of KRONOS. Adv Ther. 2020;37:1591-607. https://doi.org/10.1007/s12325-020-01266-5.

\section{Publisher's Note}

Springer Nature remains neutral with regard to jurisdictional claims in published maps and institutional affiliations.
Ready to submit your research? Choose BMC and benefit from:

- fast, convenient online submission

- thorough peer review by experienced researchers in your field

- rapid publication on acceptance

- support for research data, including large and complex data types

- gold Open Access which fosters wider collaboration and increased citations

- maximum visibility for your research: over 100M website views per year

At BMC, research is always in progress.

Learn more biomedcentral.com/submissions 